\title{
High prevalence of scrapie in a dairy goat herd: tissue distribution of disease-associated PrP and effect of $P R N P$ genotype and age
}

\author{
Lorenzo GonzÁlez $^{1 *}$, Stuart Martin ${ }^{1}$, Sílvia Sisó ${ }^{1}$, Timm KonOlD $^{2}$, \\ Angel Ortiz-Peláez ${ }^{2}$, Laura Phelan ${ }^{2}$, Wilfred Goldmann ${ }^{3}$, Paula Stewart ${ }^{3}$, \\ Ginny SAunders ${ }^{2}$, Otto WindL ${ }^{2}$, Martin JefFrey ${ }^{1}$, Stephen A.C. Hawkins ${ }^{2}$, \\ Michael DAwson ${ }^{2}$, James Hope ${ }^{1}$ \\ ${ }^{1}$ Veterinary Laboratories Agency (VLA-Lasswade), Pentlands Science Park, Penicuik, \\ Midlothian EH26 0PZ, United Kingdom \\ ${ }^{2}$ VLA-Weybridge, Addlestone, Surrey KT15 3NB, United Kingdom \\ ${ }^{3}$ Roslin Institute Neuropathogenesis Division, Roslin, Midlothian EH25 9PS, United Kingdom
}

(Received 8 April 2009; accepted 14 August 2009)

\begin{abstract}
Following a severe outbreak of clinical scrapie in 2006-2007, a large dairy goat herd was culled and 200 animals were selected for post-mortem examinations in order to ascertain the prevalence of infection, the effect of age, breed and PRNP genotype on the susceptibility to scrapie, the tissue distribution of diseaseassociated $\operatorname{PrP}\left(\operatorname{PrP}^{\mathrm{d}}\right)$, and the comparative efficiency of different diagnostic methods. As determined by immunohistochemical (IHC) examinations with Bar224 PrP antibody, the prevalence of preclinical infection was very high $(72 / 200 ; 36.0 \%)$, with most infected animals being positive for $\mathrm{PrP}^{\mathrm{d}}$ in lymphoreticular system (LRS) tissues $(68 / 72 ; 94.4 \%)$ compared to those that were positive in brain samples $(38 / 72 ; 52.8 \%)$. The retropharyngeal lymph node and the palatine tonsil showed the highest frequency of $\operatorname{PrP}^{\mathrm{d}}$ accumulation $(87.3 \%$ and $84.5 \%$, respectively), while the recto-anal mucosa-associated lymphoid tissue (RAMALT) was positive in only $30(41.7 \%)$ of the infected goats. However, the efficiency of rectal and palatine tonsil biopsies taken shortly before necropsy was similar. The probability of brain and RAMALT being positive directly correlated with the spread of $\operatorname{PrP}^{\mathrm{d}}$ within the LRS. The prevalence of infection was influenced by $P R N P$ genetics at codon 142 and by the age of the goats: methionine carriers older than 60 months showed a much lower prevalence of infection $(12 / 78 ; 15.4 \%)$ than those younger than 60 months $(20 / 42 ; 47.6 \%)$; these last showed prevalence values similar to isoleucine homozygotes of any age $(40 / 80 ; 50.0 \%)$. Two of seven goats with definite signs of scrapie were negative for $\operatorname{PrP}^{\mathrm{d}}$ in brain but positive in LRS tissues, and one goat showed biochemical and IHC features of $\operatorname{PrP}^{\mathrm{d}}$ different from all other infected goats. The results of this study have implications for surveillance and control policies for scrapie in goats.
\end{abstract}

scrapie / goat / prion disease / transmissible spongiform encephalopathy

\section{INTRODUCTION}

Scrapie is a progressive and fatal degeneration of the central nervous system (CNS) that

\footnotetext{
* Corresponding author: 1.gonzalez@vla.defra.gsi.gov. uk, this article is subject to British Crown copyright.
}

occurs naturally in small ruminants. Scrapie in sheep was the first disease to be recognized within the group of the transmissible spongiform encephalopathies (TSE), which affect several mammalian species, including man and cattle. Naturally-occurring scrapie in goats was first reported in France [5], and although 
this and other subsequent descriptions [3, 16, 34] record contact with scrapie-infected sheep, some others do not [15, 29, 34]. In most cases, the disease in goats has been reported to closely resemble that in sheep, although differences in clinical manifestation between the two species have been described [4].

Experimentally, goats are susceptible to sheep scrapie [27] and to intracerebral or oral infection with the bovine spongiform encephalopathy (BSE) agent [9]. The goat population in Europe (around 13 million), and particularly in Great Britain (around 95000 ), is considerably smaller than the sheep population (around 100 and 24 million, respectively [8]), but most European goats, unlike sheep, are kept for dairy purposes. Therefore, historically, goats are more likely than sheep to have been exposed to the BSE agent in concentrate feed; this is akin to the relatively higher BSE exposure of dairy cows compared to beef suckler cattle [32]. Indeed, the first naturally-occurring case of BSE in small ruminants was reported in a goat in France [6], and another probable goat case with BSE-like immunohistochemical (IHC) characteristics has emerged from a retrospective study in Scotland ${ }^{1}$ [19]. However, our ability to discriminate scrapie from BSE in goats is hindered by a lack of information on the range of molecular, pathological and biological characteristics of natural caprine scrapie cases.

The susceptibility to scrapie in small ruminants is controlled in part by the primary sequence of the prion protein gene (PNRP). This is well established for sheep, but less well examined for goats [11]. The most common amino acid polymorphisms in British goat breeds are at codons 127 (glycine or serine), 142 (isoleucine or methionine), 143 (histidine or arginine) and 240 (proline or serine), and an association of polymorphisms at codons 142 and 143 with susceptibility and incubation period has been reported $[1,2,10]$. A comprehensive review of the goat PNRP gene polymorphisms and their association with disease has recently been published [31].

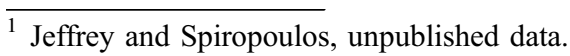

Following confirmation of scrapie in a large herd of dairy goats in the South of England, the whole herd was culled as required by the legislation in place at the time. This provided the opportunity to investigate the effect of breed, age and PNRP genotype on the prevalence of infection and tissue distribution of disease-associated $\operatorname{PrP}\left(\operatorname{PrP}^{\mathrm{d}}\right)$, to assess the efficiency of statutory testing methods in the detection of preclinical infection, and to characterize the clinical signs of the disease. These data can be used in evaluating strategies for the management and control of future TSE outbreaks in goat herds.

\section{MATERIALS AND METHODS}

\subsection{Herd and case history}

This herd was created in 1998 with the purchase of $\sim 400$ goats from a single herd. Two main additions to the herd of $\sim 100$ goatlings each time took place in 2003 and 2007. This was an intensively managed, permanently housed dairy herd, which increased in size progressively to meet the developing market for goat milk. Consequently, there was a low cull/replacement policy so, at the time of culling, it consisted of 1820 animals, 1307 of which were one year and older. Three breeds, the Anglo-Nubian $(66.0 \%)$, the Saanen $(23.0 \%)$ and the Toggenburg $(8.0 \%)$, either as pure-breeds or as crosses, accounted for $97.1 \%$ of the stock. No presence of or contact with sheep was recorded at any time since the creation of the herd to its culling.

The index case of scrapie on this holding was diagnosed in a fallen goat in 2005. After confirmation, the herd was put under movement restriction and monitored closely. Five scrapie cases were detected up until March 2006, another 20 between April 2006 and March 2007 and 50 from April 2007 until the herd was finally culled in March 2008. If only goats older than one year are considered, the annual incidence of scrapie rose significantly from $0.6 \%$ in $2005-2006$, to $2.4 \%$ in 2006-2007 and to $5.0 \%$ in 2007-2008.

When the herd was culled, a selection of 200 goats older than 12 months was made, partly on the basis of their PNRP genotype (120) and partly at random (80), for the studies detailed below. The genotype frequencies at codon 142 in these 200 goats were $40.0 \% \mathrm{II}_{142}, 46.5 .0 \% \mathrm{IM}_{142}$ and $13.5 \% \mathrm{MM}_{142}$ (I, isoleucine; M, methionine). 


\subsection{Clinical monitoring and biopsy examinations}

The 200 selected goats were transported to the Veterinary Laboratories Agency facilities in Weybridge (UK) where they were clinically examined on arrival. A short examination protocol was used to assess body condition, posture, locomotion, behaviour, presence of tremor, pruritus and vision. Repeated neurological examinations and monitoring by closed circuit television were conducted in those goats that were selected for milk collection and/or monitoring of clinical progression. Following the systematic clinical assessment protocol described elsewhere [22], animals were grouped as: 0 , clinically healthy; 1 , no signs of scrapie but of other disease; 2 , inconclusive signs (frequent rubbing, tremor or nervousness); 3 , definite signs of scrapie (evident signs of pruritus, such as a positive scratch test or progressive/extensive hair loss in the absence of visible ectoparasites, with or without tremor, ataxia or nervousness).

To assess biopsy methods for pre-clinical diagnosis, samples were taken from 62 of the 200 goats; 16 of them provided palatine tonsil biopsies, 17 provided rectal mucosa specimens, and 29 provided both. Most biopsy samples were collected between one and two months before euthanasia; exceptionally, some biopsies were obtained within one week prior to necropsy or three months before euthanasia. The methods for palatine tonsil and rectal mucosa biopsies have been described by Jeffrey et al. [17] and by González et al. [14], respectively. Biopsy samples were examined for the presence of $\operatorname{PrP}^{\mathrm{d}}$ by the same IHC method as for post-mortem samples (see below).

\subsection{Post-mortem samples and laboratory examinations}

Goats were killed by pentobarbital overdose injection and exsanguination. The following tissues were collected at post-mortem and fixed in $10 \%$ formaldehyde: sagittally-sectioned half brain, thoracic spinal cord (segments 10-12), distal ileum, palatine tonsil, spleen, nictitating membrane, medial retropharyngeal, pre-scapular, distal jejunal and pre-femoral lymph nodes, and recto-anal mucosaassociated lymphoid tissue (RAMALT).

Immunohistochemical examinations were conducted on all collected tissue samples. In the brain, routine IHC was only performed in sections of medulla oblongata at the obex and of diencephalon. The IHC protocol followed in this study for $\operatorname{PrP}^{\mathrm{d}}$ labelling, including antigen retrieval steps, was as described previously for the $\operatorname{PrP}$ antibody R486 [12]. Herein however, caprine tissue sections were incubated overnight at $27^{\circ} \mathrm{C}$ with primary antibody Bar224 (SPIbio, Montigny-le-Bretonneux, France), which binds to a globular domain epitope of PrP, and showed good performance for the detection of carpine $\operatorname{PrP}^{\mathrm{d}}$ [19]. In goats with a $\operatorname{PrP}^{\mathrm{d}}$ positive result, scrapie-BSE discriminatory IHC was carried out on a selected lymphoid tissue (usually retropharyngeal lymph node) and a brain section (usually obex). For that purpose, two serial sections were each incubated with either Bar224 or 12B2 (CVI, Lelystad, The Netherlands) antibodies; the 12B2 monoclonal antibody recognizes an epitope in the $\mathrm{N}$ terminus of $\mathrm{PrP}$ and gives no signal for intracellular $\operatorname{PrP}^{\mathrm{d}}$ in cases of experimental goat BSE [19].

In addition, following a conventional statutory testing protocol, samples of medulla oblongata caudal to the obex from all 200 goats were analyzed for $\mathrm{PrP}^{\text {res }}$ by a rapid test (TeSeE ELISA, Bio-Rad, France). ELISA positive cases were examined by discriminatory IHC with R145 (VLA, Weybridge, UK) and P4 (R-Biopharm AG, Darmstadt, Germany) antibodies, by discriminatory Western blotting with Sha31 (CEA, Saclay, France) and P4 antibodies, and for evidence of neuropil vacuolation by conventional histological examination. R145 and Sha31 antibodies recognize epitopes in the C-terminal and globular domains of PrP protein, respectively, and P4 recognizes an amino acid sequence close to that bound by 12B2 [18].

Blood or tissue samples from all 200 goats plus 62 of the 75 goats diagnosed with scrapie before the cull of the herd were taken for PCR amplification and sequencing of the whole open reading frame of the PNRP gene with primers PS-112d - TGTTTA TAGCTGATGCCACTGC and PS $+50 \mathrm{u}-\mathrm{CCCCC}$ AACCTGGCAAAGATTAAGA. Sequencing was performed on an Applied Biosystems 3130 Genetic Analyzer with the BigDye ${ }^{\circledR}$ terminator v3.1 cycle sequencing kit as per the manufacturer's protocol.

\section{RESULTS}

\subsection{Prevalence of infection and post-mortem testing efficiency}

Goats were considered scrapie-infected if at least one of the 12 tissue sections routinely examined by IHC showed specific $\operatorname{PrP}^{\mathrm{d}}$ labelling with Bar224 antibody. Based on this criterion, the frequency of infection in the 200 selected goats was $36.0 \%(72 / 200)$, and it did 
Table I. Summary of laboratory results, genotype and age distribution, and clinical status of the 72 scrapieinfected goats according to their $\operatorname{PrP}^{\mathrm{d}}$ distribution in brain and LRS tissues.

\begin{tabular}{lccc}
\hline & \multicolumn{3}{c}{ PrP $^{\mathrm{d}}$ IHC positive in } \\
\cline { 2 - 4 } $\begin{array}{l}\text { Other results } \\
\text { ELISA brain }\end{array}$ & $(0) 0(0)$ & LRS only $(n=34)$ & Brain and LRS $(n=34)$ \\
WB brain & $(0) 0(0)$ & $(0) 0(0)$ & $(56) 19(100)$ \\
Vacuolation & $(0) 0(0)$ & $(0) 0(0)$ & $(56) 19(100)$ \\
Genotype at 142 & & $(0) 0(0)$ & $(38) 13(100)$ \\
II & $(0) 0(0)$ & $(76) 26(65)$ & $(41) 14(35)$ \\
IM & $(50) 2(7)$ & $(21) 7(25)$ & $(56) 19(68)$ \\
MM & $(50) 2(50)$ & $(3) 1(25)$ & $(3) 1(25)$ \\
Age (months) & & $(38) 13(41)$ & $(47) 16(50)$ \\
$<60$ & $(75) 3(9)$ & $(62) 21(61)$ & $(53) 18(35)$ \\
$>60$ & $(25) 1(4)$ & & $(59) 20(43)$ \\
Clinical signs & & $(68) 23(50)$ & $(9) 3(50)$ \\
Normal & $(75) 3(7)$ & $(9) 3(50)$ & $(18) 6(46)$ \\
Other & $(0) 0(0)$ & $(18) 6(46)$ & $(14) 5(71)$ \\
Inconclusive & $(25) 1(8)$ & $(5) 2(29)$ & \\
Definite & $(0) 0(0)$ & &
\end{tabular}

Results are indicated as number of animals falling in each group. Percentage in brackets to the left of the number refers to the total of goats in each IHC category (brain only, LRS only or brain and LRS). Percentage in brackets to the right of the number refers to the total of goats in each set of other results, genotype, age and clinical condition (rows).

not appear to be related to breed, so that it was $42.4 \%$ for the Anglo-Nubian (50/118), 26.5\% for the Saanen (13/49), and $27.3 \%$ for the Toggenburg (6/22); these differences were not significant by Fisher's exact test. Three of 11 goats $(27.3 \%)$ of other minority breeds and crosses were also infected.

Four of the 72 positive goats showed $\operatorname{PrP}^{\mathrm{d}}$ accumulation only in the brain, 34 only in lymphoreticular system (LRS) tissues, and 34 in both brain and LRS tissues. A summary of other laboratory results, PNRP genotype and age distribution, and clinical status, in relation to this classification based on $\operatorname{PrP}^{\mathrm{d}}$ distribution in brain and LRS tissues is given in Table I. The likelihood of an IHC positive result in brain was clearly correlated to the range of distribution of $\operatorname{PrP}^{\mathrm{d}}$ in LRS tissues (Tab. II). Thus, while only 9 of 26 goats with three or fewer positive LRS tissues showed $\operatorname{PrP}^{\mathrm{d}}$ in the brain, 22 of 31 with seven or more positive LRS tissues did so; these proportions were significantly different in the Fisher's exact test $(p<0.01)$. Therefore, the wider the $\operatorname{PrP}^{\mathrm{d}}$ dissemination in
Table II. Probability of detection of $\operatorname{PrP}^{\mathrm{d}}$ in the brain in relation to the spread of infection in LRS tissues.

\begin{tabular}{lccc}
\hline Brain result & \multicolumn{3}{c}{$\begin{array}{c}\text { Positive LRS tissues out } \\
\text { of nine examined }\end{array}$} \\
\cline { 2 - 4 } & $0-3$ & $4-6$ & $7-9$ \\
\hline Positive/tested & $9^{*} / 26$ & $7 / 15$ & $22 / 31$ \\
$\%$ & 34.6 & 46.7 & 71.0 \\
Significance $^{* *}$ & A & AB & B \\
\hline
\end{tabular}

* The nine CNS positive cases associated with low $\mathrm{PrP}^{\mathrm{d}}$ in LRS tissues include the four cases without any $\mathrm{PrP}^{\mathrm{d}}$ in LRS.

** Percentage values with no letter in common are significantly different $(p<0.05)$ in the Fisher's exact test.

the LRS, the higher the likelihood of $\operatorname{PrP}^{\mathrm{d}}$ accumulating in the brain is.

Only $19(26.4 \%)$ of the 72 scrapie-infected goats were positive in the BioRad TeSeE ELISA; in other words, half of those animals that were positive in the brain by IHC were 
not detected as infected by the BioRad TeSeE ELISA. All those 19 ELISA-positive goats were amongst the cases that were IHC positive in both LRS and brain samples (Tab. I) and, on extended examinations, they showed markedly higher magnitudes of $\mathrm{PrP}^{\mathrm{d}}$ accumulation in the brain (Fig. 1a) than did IHC-positive, ELISA-negative goats (Fig. 1b). All 19 ELISA positive cases were confirmed on brain samples by IHC with R145 and by Western blotting with SHA31 antibodies; three of them were negative and another three inconclusive for spongiform pathology.

\subsection{Tissue distribution of $\operatorname{PrP}^{d}$ and ante-mortem detection of infection}

A high proportion of the scrapie-infected goats $(31 / 72 ; 43.1 \%)$ accumulated $\operatorname{PrP}^{\mathrm{d}}$ in 7 or more of the 9 LRS tissues examined, 15 $(20.8 \%)$ in 4 to 6 LRS tissues, $13(18.1 \%)$ in 2 or 3 LRS samples, and $9(12.5 \%)$ were IHC positive in a single LRS tissue. $\operatorname{PrP}^{\mathrm{d}}$ is a marker of TSE infection and this range of $\operatorname{PrP}^{\mathrm{d}}$ distribution suggests that infection in the herd was at different stages in different animals. Moreover, there was an association between the spread of infection within the LRS and the magnitude of $\operatorname{PrP}^{\mathrm{d}}$ accumulation in the specific tissues involved. Thus, in those cases in which a single LRS tissue was affected, the amounts of $\operatorname{PrP}^{\mathrm{d}}$ were very small, often within a few or even single cells resembling tingible body macrophages (Fig. 2a), while prominent $\mathrm{PrP}^{\mathrm{d}}$ deposits associated with both follicular dendritic cells and tingible body macrophages (Fig. 2b) were frequent in those cases in which the number of LRS tissues affected was also high.

Within the 72 scrapie-infected goats, the medial retropharyngeal lymph node (62/71 positive samples; $87.3 \%$ ) and the palatine tonsil $(60 / 71 ; 84.5 \%)$ showed a significantly higher frequency of $\operatorname{PrP}^{\mathrm{d}}$ accumulation than all other LRS tissues $(p<0.05)$. In descending order, the others were the distal jejunal lymph node $(44 / 72 ; 61.1 \%)$, the nictitating membrane (43/ $72 ; 59.7 \%$ ), and the pre-scapular lymph node (39/72; 54.2\%), the Peyer's patches (33/71; $46.5 \%)$, the pre-femoral lymph node $(33 / 72$; $45.8 \%)$, the RAMALT $(30 / 72 ; 41.7 \%)$ and the spleen $(27 / 72 ; 37.5 \%)$. These last two tissues showed a significantly lower rate of $\mathrm{PrP}^{\mathrm{d}}$ accumulation than the distal jejunal lymph node $(p<0.05)$.

As with the brain, the frequency of $\operatorname{PrP}^{\mathrm{d}}$ accumulation in RAMALT samples taken at post-mortem was correlated to the overall LRS involvement (Tab. III). Thus, the probability of a positive result in the RAMALT increased from an overall $41.7 \%(30 / 72)$ to $69.0 \%$ (29/42), $75.0 \%(27 / 36)$ or $84.0 \%(21 /$ 25), for animals with more than 4,5 or $6 \mathrm{PrP}^{\mathrm{d}}$ positive LRS tissues - other than the RAMALT itself -, respectively. A further assessment of the efficiency of $\operatorname{PrP}^{\mathrm{d}}$ detection in the RAMALT as a means of identifying preclinical carriers of infection comes from the results of the biopsies performed before the goats were culled. A total of 54 biopsies (26 of RAMALT and 28 of palatine tonsil) were taken from 36 goats that were TSE negative at post-mortem examinations, and none of them provided positive results (100\% specificity). Judged on 26 scrapie-positive goats on post-mortem, the sensitivity of tonsil biopsies was $52.6 \%(10 / 19)$ and that of RAMALT biopsies was $40.0 \%$ (8/ $20)$; the difference was not statistically significant. All eight goats with positive RAMALT biopsy were also $\mathrm{PrP}^{\mathrm{d}}$ positive in rectal mucosa taken at post-mortem; only one goat showing $\operatorname{PrP}^{\mathrm{d}}$ accumulation in this tissue sample at post-mortem was negative in biopsy (only two follicles present in the specimen). Equally, all 10 goats with positive tonsil biopsy result showed $\operatorname{PrP}^{\mathrm{d}}$ accumulation in this sample taken at post-mortem; however, six goats with positive tonsil at post-mortem had provided negative biopsy results $(1,7,9,9,16$ and 30 follicles present, respectively).

\subsection{Effect of age and $P N R P$ genotype on scrapie prevalence}

The age of the infected goats (67.5 months, 95\% CI 61.3-73.6) was significantly lower $(p<0.05$, Mann-Whitney test) than that of the non-infected animals (76.5 months, 95\% CI 71.7-81.3). These observations were confirmed by the comparative analysis of two age cohorts (Tab. IV), so that the Fisher's exact 

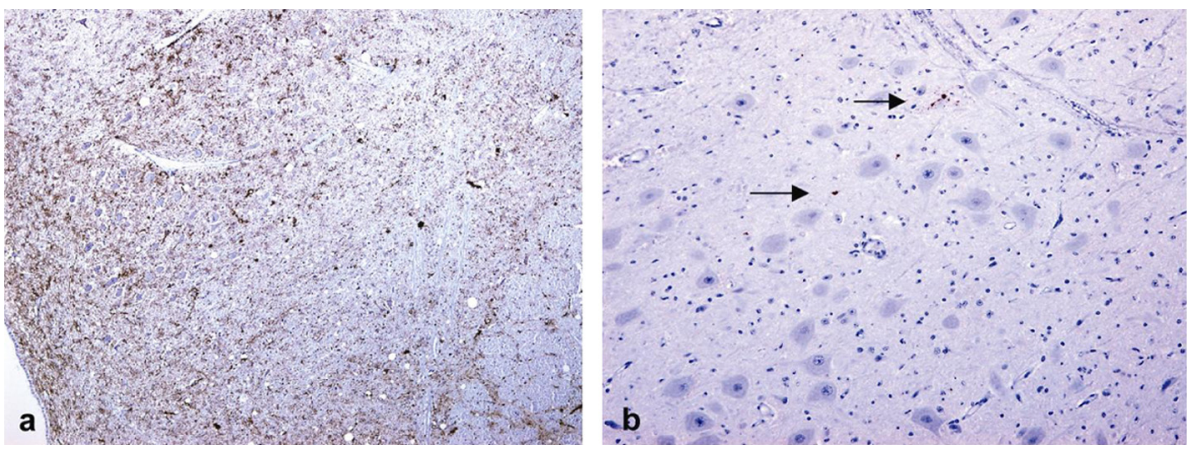

Figure 1. Accumulation of $\operatorname{PrP}^{\mathrm{d}}$ in the medulla of scrapie infected goats. (a) Abundant deposits of abnormal protein in the neuropil of the dorsal motor nucleus of the vagus nerve (DMNV) and adjacent structures, with occasional heavily labelled coalescing $\operatorname{PrP}^{\mathrm{d}}$ aggregates; serial samples of caudal medulla provided positive ELISA results. IHC with Bar224 PrP antibody and haematoxylin counterstaining, $\times 10$. (b) Trace extracellular deposits (arrows) in the DMNV of a clinically healthy goat that provided negative ELISA results in serial samples of caudal medulla. IHC with Bar224 PrP antibody and haematoxylin counterstaining, $\times 20$.
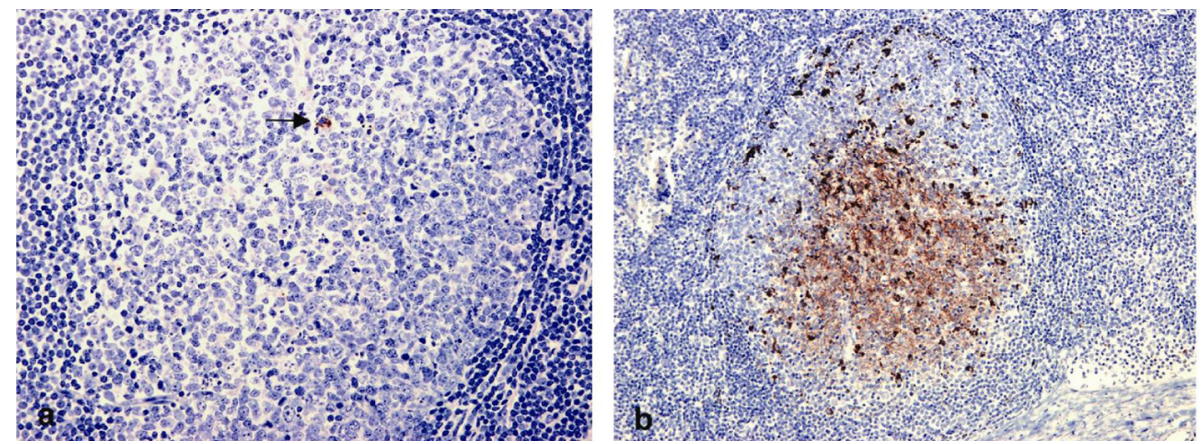

Figure 2. Accumulation of $\operatorname{PrP}^{\mathrm{d}}$ in lymphoreticular tissues (retropharyngeal lymph nodes) of scrapie infected goats. (a) Intracellular deposits (arrow) in single or few cells in the light zone of a single secondary follicle of a single lymph node were the only indication of infection in some goats. IHC with Bar224 PrP antibody and haematoxylin counterstaining, $\times 20$. (b) In some other animals, accumulation of $\operatorname{PrP}^{\mathrm{d}}$ was widespread and severe with abundant aggregates associated with follicular dendritic cells and tingible body macrophages. IHC with Bar224 PrP antibody and haematoxylin counterstaining, $\times 10$.

test indicated significant differences $(p=0.004)$ in scrapie prevalence between the youngest $(<60$ months, 50.8\%) and the oldest $(>60$ months, $29.2 \%$ ) groups. The split of ages into younger and older than 60 months was chosen because no goats aged between 54 and 65 months were included within the 200 examined.

Homozygosity for isoleucine at codon 142 $\left(\mathrm{II}_{142}\right)$ was linked to scrapie infection. These goats showed a significantly higher prevalence of infection $(40 / 80 ; 50.0 \%)$ than methionine homozygotes $\left(\mathrm{MM}_{142} ; 4 / 27 ; 14.8 \%\right)$, and than $\mathrm{IM}_{142}$ heterozygotes $(28 / 93 ; 30.1 \%)$. The Fisher's exact test showed a significantly higher probability of scrapie infection for the $\mathrm{II}_{142}$ group when compared to the $\mathrm{IM}_{142}$ group $(p<0.01)$, to the $\mathrm{MM}_{142}$ group $(p=0.001)$, and to the two of them together $(p<0.001)$. Significant differences were not found between 
Table III. Probability of detection of $\mathrm{PrP}^{\mathrm{d}}$ in the RAMALT in relation to the spread of infection in LRS tissues.

\begin{tabular}{lccc}
\hline $\begin{array}{l}\text { RAMALT } \\
\text { result }\end{array}$ & \multicolumn{3}{c}{$\begin{array}{c}\text { Positive LRS tissues out of } \\
\text { 8 examined }\end{array}$} \\
\cline { 2 - 4 } & $0-4$ & $5-6$ & $7-8$ \\
\hline Positive/tested & $1 / 30$ & $8 / 17$ & $21 / 25$ \\
$\%$ & 3.3 & 47.1 & 84.0 \\
Significance & A & B & C \\
\hline${ }^{*}$ Excluding the RAMALT itself. \\
${ }^{* * *}$ Percentage values with no letter in common are \\
significantly different $(p<0.05)$ in the Fisher's exact \\
test.
\end{tabular}

Table IV. Prevalence of scrapie infection in relation to the age and genotype of the goats at codon 142 of PrP.

\begin{tabular}{lccccc}
\hline Scrapie result & \multicolumn{4}{c}{$\begin{array}{c}\text { Age cohorts (months) and 142 } \\
\text { polymorphism }\end{array}$} \\
\cline { 2 - 4 } \cline { 2 - 3 } \cline { 5 - 6 } & \multicolumn{2}{c}{$<60$} & & \multicolumn{2}{c}{$>60$} \\
\cline { 2 - 3 } \cline { 5 - 6 } & II & IM/MM & & II & IM/MM \\
\hline Positive/tested & $12 / 21$ & $20 / 42$ & & $28 / 59$ & $12 / 78$ \\
Significance $^{*}$ & 57.1 & 47.6 & & 47.4 & 15.4 \\
\hline
\end{tabular}

Percentage values with no letter in common are significantly different $(p<0.05)$ in the Fisher's exact test.

the heterozygotes and the $\mathrm{MM}_{142}$ goats $(p=0.1)$, indicating a protective, though incomplete, effect of methionine at this position. Genotypes at this codon were available for 62 of the 75 scrapie cases confirmed in this herd prior to the cull. When these were added to the post-cull data, the link between homozygosity for isoleucine at codon 142 and infection became even more evident, as 55 of those 62 cases $(88.7 \%)$ were $\mathrm{II}_{142}$ homozygotes and the other 7 were $\mathrm{IM}_{142}$ heterozygotes.

The effect of the genotype at codon 142 on the prevalence of scrapie appeared to be modulated by the age of the animals (Tab. IV). Thus, in the group younger than 60 months, isoleucine homozygotes and methionine carriers
( $\mathrm{IM}_{142}$ and $\mathrm{MM}_{142}$ together) showed a very similar probability of infection $(57.1 \%$ and $47.6 \%$, respectively); in contrast, $\mathrm{II}_{142}$ goats older than 60 months were much more likely to be infected than methionine carriers $(47.4 \%$ and $15.4 \%$, respectively; $p<0.0001)$. Moreover, while the probability of infection in isoleucine homozygotes did not vary with age, methionine carriers younger than 60 months showed a significantly higher $(p=0.0002)$ prevalence of infection (47.6\%) than older animals of the same genotypes $(15.4 \%)$. The overall age results provided at the beginning of this section were therefore influenced by the fact that two thirds of the youngest goat group were methionine carriers, while genotypes were more balanced in the oldest age group.

\subsection{Relationship between infection and clinical disease}

Seven of the 200 selected goats were identified as showing definite signs of scrapie (group 3). Thirty animals had inconclusive signs (group 2), 20 displayed signs of other unrelated conditions (group 1) and the rest (143) were considered healthy (group 0). All seven clinical scrapie suspects were isoleucine homozygotes at codon 142, and were confirmed as infected by IHC, although two of them, both over 96 months of age, lacked detectable $\operatorname{PrP}^{\mathrm{d}}$ in the brain, and were positive only in LRS tissues. The other five showed prominent and widespread $\operatorname{PrP}^{\mathrm{d}}$ aggregates in the brain and were BioRad TeSeE ELISA positive; three of them were between 47 and 53 months old and two were older than 96 months.

Differences in prevalence of infection were absent between groups 0,1 and 2, with figures of $32.1 \%, 30.0 \%$ and $43.3 \%$, respectively. These values were, however, significantly lower than the $100 \%$ observed in the scrapie suspects.

\subsection{Discriminatory testing for scrapie and BSE}

All but one of the infected goats provided a $\mathrm{PrP}^{\mathrm{d}}$ epitope mapping signature of classical scrapie: intracellular $\operatorname{PrP}^{\mathrm{d}}$ aggregates, both in LRS tissues and in the brain (when present), 
were immunolabelled with similar intensity by Bar224 and 12B2 antibodies. For those goats that were positive in the brain and gave a positive Bio-Rad ELISA result, these findings were corroborated by IHC with R145 and P4 antibodies and by Western blot with SHA31 and P4 antibodies.

One ELISA-positive, clinically unremarkable, eight year-old Anglo-Nubian goat, homozygous for isoleucine at codon 142, provided a protease-resistant PrP Western blot pattern different from the rest, with a lower molecular weight of the unglycosylated band and a markedly reduced signal with $\mathrm{mAb} \mathrm{P} 4$. In IHC, while extracellular $\operatorname{PrP}^{\mathrm{d}}$ aggregates were revealed with both Bar224 and 12B2 antibodies (Figs. $3 \mathrm{a}$ and $3 \mathrm{~b}$ ), intraneuronal and intramicroglial $\operatorname{PrP}^{\mathrm{d}}$ deposits in thalamic nuclei, and in the accessory cuneate, trigeminal and olivary nuclei of the obex, were prominent with R145 and Bar224 antibodies but were not revealed at all with either P4 or 12B2 monoclonal antibodies (Figs. 3c and 3d). Therefore, both biochemical and intracellular truncation patterns of $\mathrm{PrP}^{\mathrm{d}}$ in brain tissue of this goat were compatible with experimental BSE in small ruminants $[20,30]$. However, in the retropharingeal lymph node, $\operatorname{PrP}^{\mathrm{d}}$ aggregates within the cytoplasm of tingible body macrophages were labelled with both Bar224 and 12B2 (Figs. 3e and 3f); this was in contrast with an experimental BSE control goat lymph node examined in parallel, which showed no intracellular $\mathrm{PrP}^{\mathrm{d}}$ with 12B2. Also, all Western blot features, including the SHA31/P4 signal ratio, of a sample of retropharyngeal lymph node of this goat were those of scrapie. Moreover, further IHC examinations in brain sections from this animal, showed no intracellular immunolabelling with antibodies 9A2 and 1E4, which recognize epitopes more C-terminally located than that of 12B2. Other antibodies such as 521,505 or IDPrP4, which recognize epitopes in the same regions as $9 \mathrm{~A} 2$ and $1 \mathrm{E} 4$, produced strong intraneuronal signal in cases of experimental caprine BSE [19].

Routine IHC examinations performed in the brain of the 200 goats did not show any $\operatorname{PrP}$ accumulation suggestive, either morphologically or topographically, of Nor98/atypical scrapie.

\section{DISCUSSION}

\subsection{Implications for surveillance of goat TSE}

Scrapie in this goat herd reached a high prevalence of infection and a high incidence of clinical disease. This may be due to the intensive management system of this commercial dairy herd, where animals were kept at high density indoors and where husbandry practices, such as pooling of colostrum and milk for feeding to kids and the use of group kidding pens were common. The extensive range of tissues examined and the high level of scrutiny allowed detection of cases where $\operatorname{PrP}^{\mathrm{d}}$ was restricted to a low number of cells within a single tissue; it is thus possible that such low levels of immunoreactivity may have been missed in some goats. Moreover, the selection of the 200 goats was biased against isoleucine homozygotes $(40.0 \%$ amongst the 200 versus $50.0 \%$ in a random sample of 298 goats analyzed in 2007; results not shown). Therefore, the scrapie prevalence figure obtained may underestimate the true level of infection.

While clinical signs of disease were absent in several animals with widespread $\operatorname{PrP}^{\mathrm{d}}$ in brain, definite signs were observed repeatedly in two animals without detectable $\operatorname{PrP}^{\mathrm{d}}$ in their brains. Lack of correlation of brain $\mathrm{PrP}^{\mathrm{d}}$ and clinical signs has been reported in sheep scrapie [20], and the absence of both indicators of disease in almost half of infected goats in this study underlines the limits of their use in monitoring herds for infection.

Current surveillance programmes, where only samples of brain are examined [26], will clearly provide a poor estimate of the prevalence of scrapie, at least in flocks or herds with high levels of infection. Previous work had suggested that examination of LRS tissues in addition to brain provides better estimates of scrapie infection in sheep $[13,25,28]$. Our results are a noticeable example that this is also the case in goats and that, in agreement with those earlier sheep studies, the medial retropharyngeal lymph node would be the sample of choice, together with the obex, for post-mortem surveillance. The relative insensitivity of the rapid screening test used for statutory purposes in this study compared to IHC was probably due to the 

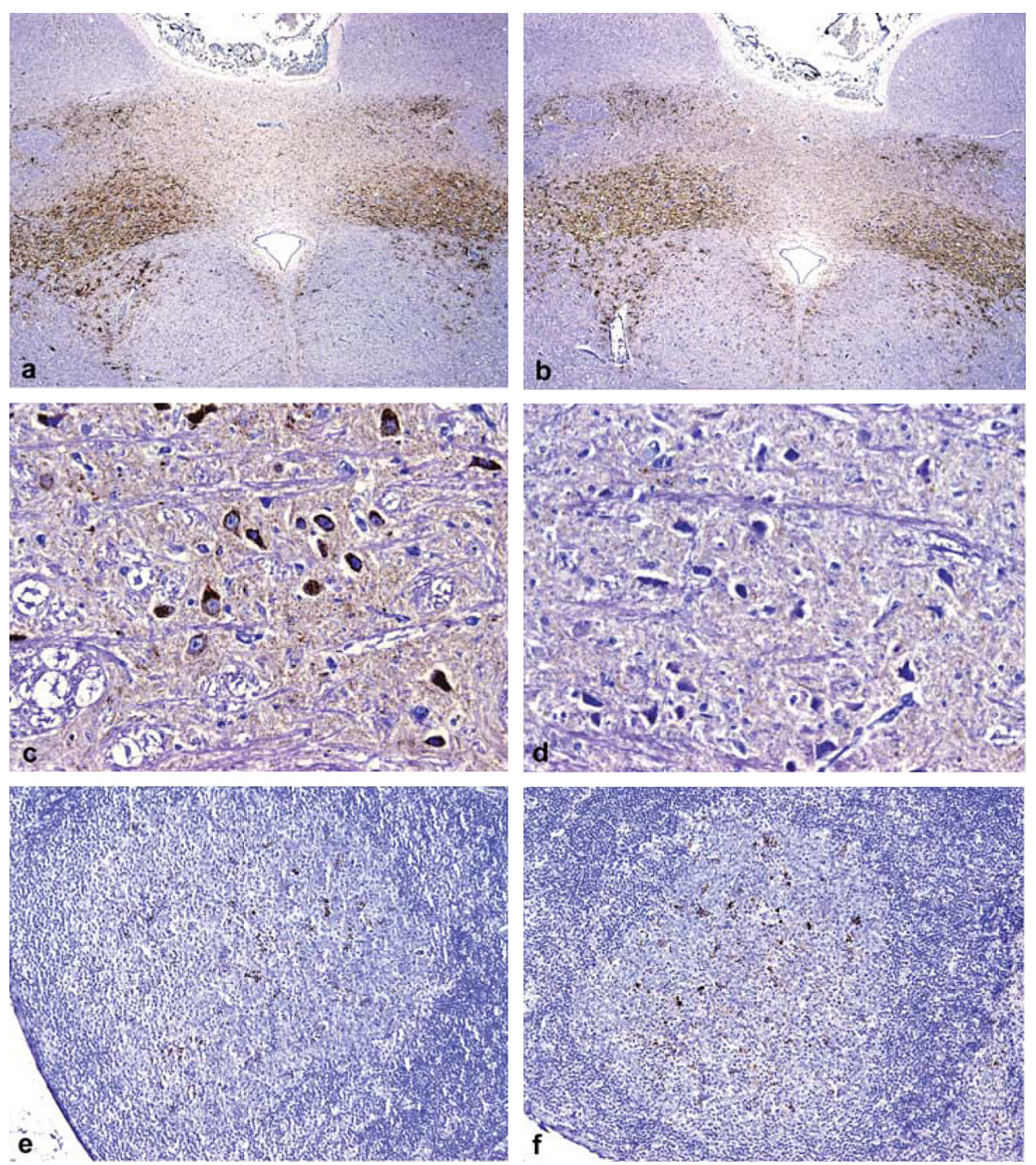

Figure 3. Discriminatory immunohistochemistry on an unusual goat TSE case. Extracellular accumulation of $\operatorname{PrP}^{\mathrm{d}}$ in the brain was revealed with both Bar224 (a) and 12B2 (b) PrP antibodies. IHC and haematoxylin counterstaining, $\times 4$. Intraneuronal $\mathrm{PrP}^{\mathrm{d}}$ deposits in the olivary nucleus were prominent when labelled with Bar224 (c) but absent when incubated with 12B2 antibody (d). IHC and haematoxylin counterstaining, $\times 20$. In the retropharyngeal lymph node, however, intracellular $\operatorname{PrP}^{\mathrm{d}}$ deposits were labelled with both bar224 (e) and $12 \mathrm{~B} 2$ (f) antibodies. IHC and haematoxylin counterstaining, $\times 10$.

very low level of $\operatorname{PrP}^{\mathrm{d}}$ deposits found, restricted to the dorsal motor nucleus of the vagus nerve in some animals, together with the sub-optimal site of sampling - caudal to the obex - for rapid testing, imposed by the design of the study. In addition, it can also be considered that the
BioRad TeSeE assay used for statutory diagnosis in these goats can be less sensitive than other "rapid tests" such as the BioRad TeSeE sheep and goats or the Idexx Herd Chek.

Post-mortem detection of $\operatorname{PrP}^{\mathrm{d}}$ in the RAMALT was achieved in a low percentage 
$(41.7 \%)$ of positive goats. This was unexpected considering previous figures obtained in preclinical sheep scrapie $(86.0 \%,[13])$, and similar ones in chronic wasting disease of cervids [21, 33]. Whether this is a feature of goat scrapie in general or a peculiarity of this outbreak is unknown but, in any case, the frequency of detection of $\mathrm{PrP}^{\mathrm{d}}$ in the RAMALT rises to $84.0 \%$ when goats with widespread LRS involvement - but still at preclinical stages are considered. Encouragingly, rectal biopsies for ante-mortem diagnosis of goat scrapie taken shortly before necropsy gave only slightly, nonsignificantly inferior results $(40.0 \%)$ to those of tonsil biopsies (52.6\%). The lower efficiency of tonsil biopsies when compared to post-mortem values $(84.5 \%)$ can be due to the proportion of positive follicles in some tonsil samples being very small, together with the low numbers of follicles in some biopsy specimens. These sensitivity data can be used to correct estimates of prevalence of infection based on RAMALT or palatine tonsil ante-mortem testing to control future goat scrapie outbreaks.

\subsection{Risk factors for high prevalence of infection}

Following earlier epidemiological observations reporting a higher risk of scrapie amongst lambs sucking from infected dams than in those reared artificially [7], evidence of scrapie infectivity in sheep milk [24] and of transmission via sheep milk and/or colostrum has been documented [23]. Sharing of milk/colostrum and cross-fostering of animals were frequent practices within this commercial dairy herd and may have contributed to the rapid increase of disease after the diagnosis of the index case in 2005. Around $1000 \mathrm{~L}$ of milk were collected from scrapie-infected goats of this herd, and transmission studies are planned to determine the infectivity of goats milk.

The analysis of the influence of the polymorphism at codon 142 of the caprine PrP has highlighted some interesting points. Protracted incubation periods, but no protection in terms of attack rate, were observed in methionine carriers at this codon challenged by parenteral routes with a diversity of TSE agents [10], while in this study of natural infection, those polymorphisms appeared to influence the susceptibility to infection. Thus, methionine carriers showed a lower overall prevalence than isoleucine homozygotes, but when doing a combined analysis together with the age, that effect was only seen in goats older than 60 months. One possible interpretation (Fig. 4) is that at the beginning of the scrapie epidemic, perhaps until 2003 (5 years before the cull took place), the pressure of infection was relatively low and mostly isoleucine homozygotes became infected; this could explain the low prevalence of scrapie amongst $\mathrm{M}_{142}$ carriers older than 60 months $(15.4 \%)$ compared to isoleucine homozygotes of the same age range (47.4\%). At that time, and probably helped by the intensive management imposed, the prevalence of infection would have started to rise exponentially, exerting progressively a pressure that made young $\mathrm{M}_{142}$ carriers no longer resistant to infection; this would have resulted in similar prevalence values amongst $\mathrm{M}_{142}$ carriers $(47.6 \%)$ and $\mathrm{II}_{142}$ goats $(57.1 \%)$ younger than 60 months at the time of culling. However, a higher pressure of infection in recent years should have also resulted in a higher prevalence amongst young isoleucine homozygotes, which was not the case. Two possible explanations are (i) the disappearance from the herd, due to clinical disease in 2006 and 2007, of a high number of $\mathrm{II}_{142}$ goats, which would have fitted into that young age group, and (ii) that $\sim 60 \%$ prevalence amongst isoleucine homozygotes is the highest figure that can be reached in natural conditions, whatever the pressure of infection may be. In any case, it would appear that intensive management practices leading to high pressure of infection may compromise any attempt to control scrapie in dairy goat herds by genetic selection on codon 142; whether or not this also applies to polymorphisms at other codons affecting scrapie susceptibility [31] remains to be established.

\subsection{Strain diversity}

The detection in this goat herd of a TSE case with unusual IHC and biochemical features raised initial concerns, as BSE could not be excluded. However, further IHC examinations 


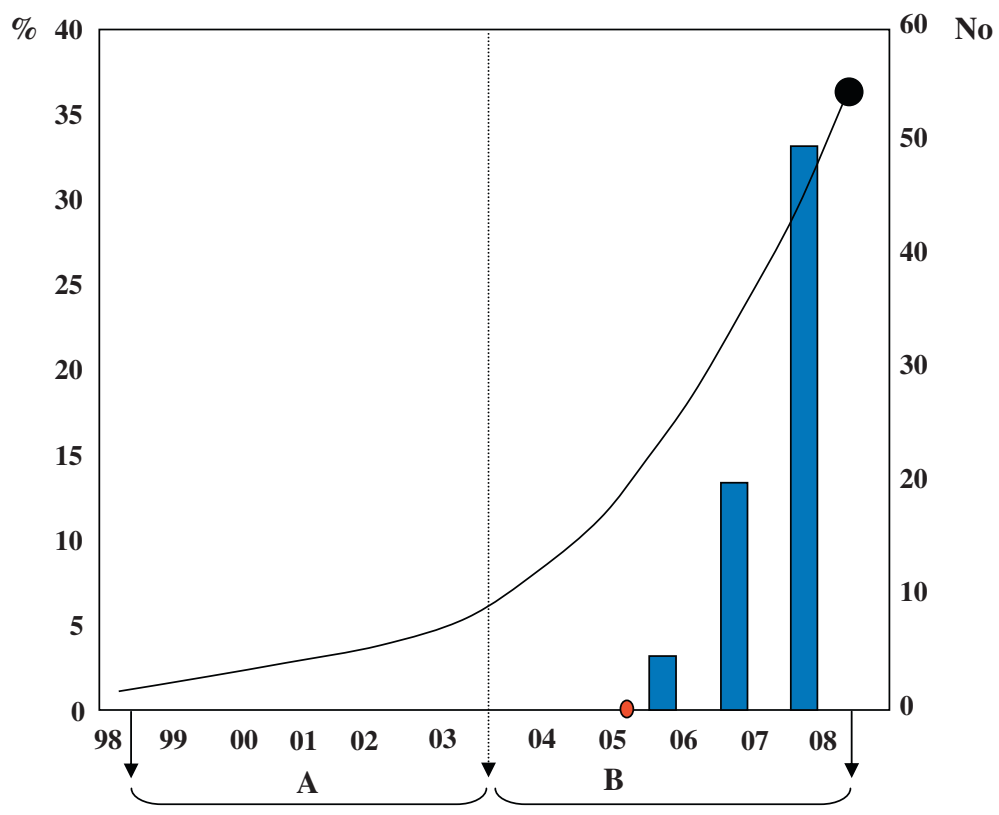

Figure 4. Diagrammatic representation of the hypothetical dynamics of scrapie infection in the herd. Left axis, prevalence of infection. Left arrow, purchase of the original herd. Right axis, number of scrapie cases recorded prior to the cull, which are represented as blue bars; red circle, index scrapie case. Right arrow, time of cull. X-axis, years. Exponential line, hypothetical increase of scrapie prevalence in the herd as a result of mounting pressure of infection, until reaching the $36.0 \%$ figure observed in the 200 goats examined (see text). Middle arrow dividing periods A and B is set in 2003 to accommodate for the age split (younger and older than 60 months) used to analyse the effect of age on prevalence. During period A, the prevalence of infection would have been low, mostly involving the most susceptible $\mathrm{II}_{142}$ goats; clinical cases would have been very few, unnoticed and undiagnosed. Therefore, most (88.7\%) of the 75 scrapie cases detected later (blue bars) were in $\mathrm{II}_{142}$ goats (see text). Also, at the time of the cull, the prevalence of infection amongst goats older than 60 months (i.e. born during period A) was significantly higher in $\mathrm{II}_{142}$ homozygotes than in methionine carriers ( $47.4 \%$ and $15.1 \%$, respectively, see Tab. IV). Raising pressure of infection during period $\mathrm{B}$ would have made young methionine carriers to become susceptible to infection, so that, at the time of the cull, methionine carriers younger than 60 months (i.e. born during period B) showed similar prevalence of infection than $\mathrm{II}_{142}$ homozygotes, and significantly higher than methionine carriers born during period A (see Tab. IV). It is assumed that scrapie infection was already present in the herd when originally purchased in 1998, as it is highly unlikely that neither prevalence of infection at the time of culling (early 2008) nor initial detection of clinical scrapie cases in 2005 could have resulted from the incorporation of 100 goatlings to the herd in 2003.

of the brain and lymphoid tissues revealed features different from those of goat BSE, at least from that experimentally reproduced with bovine inoculum by the intracerebral route [19]. This animal may represent a natural case of mixed infection with two or more strains and will be further investigated by mouse bioassay to improve our understanding of the diversity of goat TSE. Being able to identify different natural goat TSE strains, particularly $\mathrm{BSE}$, is an important issue since goat products, in particular milk, yoghurt and cheese, could be potential threats to human health.

Acknowledgements. The authors would like to thank Defra's (project SE 1956) and FSA's (project 
MO3065) financial support, the field staff of the Animal Health $(\mathrm{AH})$ agency for supervising the cull and selecting the goats for transport to the VLA, and the Scrapie Flocks team in AH Central Operations for their co-ordinating activities. We are grateful to VLA staff of the Animal Services Unit, Pathology, Molecular Pathogenesis and Genetics and Laboratory Services for their help and support with animal upkeeping, necropsies and TSE statutory diagnosis examinations. Antibody 12B2 was generously donated by J. Langeveld (CVI, Lelystad, The Netherlands). Lynn Fairlie, Ann Dunachie, Maria Oliva and Caroline Goodsir are acknowledged for their excellent work in processing formalin-fixed tissues and performing immunohistochemistry.

\section{REFERENCES}

[1] Barillet F., Mariat D., Amigues Y., Faugeras R., Caillat H., Moazami-Goudarzi K., et al., Identification of seven haplotypes of the caprine $\operatorname{Pr} P$ gene at codons 127, 142, 154, 211, 222 and 240 in French Alpine and Saanen breeds and their association with classical scrapie, J. Gen. Virol. (2009) 90:769-776.

[2] Billinis C., Panagiotidis C.H., Psychas V., Argyroudis S., Nicolaou A., Leontides S., et al., Prion protein gene polymorphisms in natural goat scrapie, J. Gen. Virol. (2002) 83:713-721.

[3] Brotherson J.C., Renwick C.C., Stamp J.T., Zlotnik I., Pattison I.H., Spread of scrapie by contact to goats and sheep, J. Comp. Pathol. Ther. (1968) 78:9-17.

[4] Capucchio M.T., Guarda F., Pozzato N., Coppolino S., Caracappa S., Di Marco V., Clinical signs and diagnosis of scrapie in Italy: a comparative study in sheep and goats, J. Vet. Med. A Physiol. Pathol. Clin. Med. (2001) 48:23-31.

[5] Chelle P.-L., Un cas de tremblante chez la chèvre, Bull. Acad. Vet. Fr. (1942) 15:294-295.

[6] Eloit M., Adjou K., Coulpier M., Fontain J.-J., Hamel R., Lilin T., et al., BSE agent signatures in a goat, Vet. Rec. (2005) 156:523-524.

[7] Elsen J.M., Amigues Y., Schelcher F., Ducroc V., Andréoletti O., Eychenne F., et al., Genetic susceptibility and transmission factors in scrapie: detailed analysis of an epidemic in a closed flock of Romanov, Arch. Virol. (1999) 144:431-445.

[8] Eurostat, EU sheep and goat population in December 2007 and production forecasts for 2008, Eurostat statistics in focus 67/2008 (2008) http:// www.eds-destatis.de/de/downloads/sif/sf_08_067.pdf.

[9] Foster J., Hope J., Fraser H., Transmission of bovine spongiform encephalopathy to sheep and goats, Vet. Rec. (1993) 133:339-341.
[10] Goldmann W., Martin T., Foster J., Hughes S., Smith G., Hughes K., et al., Novel polymorphisms in the caprine PrP gene: a codon 142 mutation associated with scrapie incubation period, J. Gen. Virol. (1996) 77:2885-2891.

[11] Goldmann W., $\operatorname{Pr} P$ genetics in ruminant transmissible spongiform encephalopathies, Vet. Res. (2008) 39:30.

[12] González L., Martin S., Begara-McGorum I., Hunter N., Houston F., Simmons M., Jeffrey M., Effects of agent strain and host genotype on PrP accumulation in the brain of sheep naturally and experimentally affected with scrapie, J. Comp. Pathol. (2002) 126:17-29.

[13] González L., Dagleish M.P., Bellworthy S.J., Sisó S., Stack M.J., Chaplin M.J., et al., Postmortem diagnosis of preclinical and clinical scrapie in sheep by the detection of disease-associated PrP in their rectal mucosa, Vet. Rec. (2006) 158:325-331.

[14] González L., Dagleish M.P., Martin S., Dexter G., Steele P., Finlayson J., Jeffrey M., Ante-mortem diagnosis of preclinical sheep scrapie by immunohistochemical examination of rectal biopsy samples, Vet. Rec. (2008) 162:397-403.

[15] Harcourt R.A., Anderson M.A., Naturallyoccurring scrapie in goats, Vet. Rec. (1974) 94:504

[16] Hourrigan J.L., Klingsporn A.L., McDaniel H.A., Riemenschneider M.N., Scrapie in a goat, J. Am. Vet. Med. Assoc. (1969) 154:538-539.

[17] Jeffrey M., Martin S., Thomson J.R., Dingwall W.S., Begara-McGorum I., González L., Onset and distribution of tissue PrP accumulation in scrapieaffected Suffolk sheep as demonstrated by sequential necropsies and tonsillar biopsies, J. Comp. Pathol. (2001) 125:48-57.

[18] Jeffrey M., González L., Chong A., Foster J., Goldmann W., Hunter N., Martin S., Ovine infection with the agents of scrapie (CH1641 Isolate) and bovine spongiform encephalopathy: immunochemical similarities can be resolved by immunohistochemistry, J. Comp. Pathol. (2006) 134:17-29.

[19] Jeffrey M., Martin S., González L., Foster J., Langeveld J.P.M., van Zijderveld F.G., et al., Immunohistochemical features of $\operatorname{PrP}^{\mathrm{d}}$ accumulation in natural and experimental goat transmissible spongiform encephalopathies, J. Comp. Pathol. (2006) 134:171-181.

[20] Jeffrey M., González L., Classical sheep transmissible spongiform encephalopathies: pathogenesis, pathological phenotypes and clinical disease, Neuropathol. Appl. Neurobiol. (2007) 33:373-394.

[21] Keane D.P., Barr D.J., Bochsler P.N., Hall S.M., Gidlewski T., O’Rourke K.I., et al., Chronic wasting 
disease in a Wisconsin white-tailed deer farm, J. Vet. Diagn. Invest. (2008) 20:698-703.

[22] Konold T., Bone G., Vidal-Díez A., Tortosa R., Davis A., Dexter G., et al., Pruritus is a common feature in sheep infected with the BSE agent, BMC Vet. Res. (2008) 4:16.

[23] Konold T., Moore S.J., Bellworthy S.J., Simmons H.A., Evidence of scrapie transmission via milk, BMC Vet. Res. (2008) 4:14.

[24] Lacroux C., Simon S., Benestad S.L., Maillet S., Mathey J., Lugan S., et al., Prions in milk from ewes incubating natural scrapie, PLoS Pathog. (2008) 4:e1000238.

[25] Langeveld J.P.M., Jacobs J.G., Erkens J.H.F., Bossers A., van Zijderveld F.G., van Keulen L.J.M., Rapid and discriminatory diagnosis of scrapie and BSE in retro-pharyngeal lymph nodes of sheep, BMC Vet. Res. (2006) 2:19.

[26] Morignat E., Cazeau G., Bicabe A.-G., Vinard J.-L., Bensick A., Madec J.-Y., et al., Estimates of the prevalence of transmissible spongiform encephalopathies in sheep and goats in France in 2002, Vet. Rec. (2006) 158:683-687.

[27] Pattison I.H., Millson G.C., Experimental transmission of scrapie to goats and sheep by the oral route, J. Comp. Pathol. (1961) 71:171-176.

[28] Reckzeh C., Hoffmann C., Bushmann A., Buda S., Budras K.-D., Reckling K.-F., et al., Rapid testing leads to the underestimation of the scrapie prevalence in an affected sheep and goat flock, Vet. Microbiol. (2007) 123:320-327.
[29] Sofianidis G., Psychas V., Billinis C., Spyrou V., Argyroudis S., Papaioannou N., Vlemmas I., Histopathological and immunohistochemical features of natural goat scrapie, J. Comp. Pathol. (2006) 135:116-129.

[30] Stack M.J., Chaplin M.J., Clark J., Differentiation of prion protein glycoforms from naturally occurring sheep scrapie, sheep-passaged scrapie strains (CH1641 and SSBP1), bovine spongiform encephalopathy (BSE) cases and Romney and Cheviot breed sheep experimentally inoculated with BSE using two monoclonal antibodies, Acta Neuropathol. (2002) 104:279-286.

[31] Vaccari G., Panagiotidis C., Acín C., Peletto S., Berillet F., Acutis P., et al., Atate-of-the-art review of goat TSE in the European Union, with special emphasis on PRNP genetics and epidemiology, Vet. Res. (2009) 40:48.

[32] Wilesmith J.W., Ryan J.B.M., Stevenson M.A., Morris S.R., Pfeiffer D.U., Lin D., et al., Temporal aspects of the epidemic of bovine spongiform encephalopathy in Great Britain: holding-associated risk factors for the disease, Vet. Rec. (2000) 147: 319-325.

[33] Wolfe L.L., Spraker T.R., González L., Dagleish M., Sirochman T.M., Brown J.C., et al., $\operatorname{PrP}^{\mathrm{CWD}}$ in rectal lymphoid tissue of deer (Odocoileus spp.), J. Gen. Virol. (2007) 88:2078-2082.

[34] Wood J.N.L., Done S.H., Pritchard G.C., Wooldridge M.J.A., Natural scrapie in goats: case histories and clinical signs, Vet. Rec. (1992) 131:6-68. 